\title{
Cursos de Extensão de Lógica de Programação para Alunos do Ensino Fundamental usando Learning-by- doing: Um relato de experiência
}

\author{
Lucas Buligon Antunes \\ IFPR - Instituto Federal do Paraná \\ Quedas do Iguaçu, Brasil \\ lukasbuligonantunes@gmail.com
}

\author{
Odair Moreira de Souza \\ IFPR - Instituto Federal do Paraná \\ Quedas do Iguaçu, Brasil \\ odair.desouza@ifpr.edu.br
}

\begin{abstract}
A new educational conjuncture has been tracing due to the current technological evolution and research demonstrates the need for new didactic approaches for teaching computer programming. The aim of this study is to present the approaches applied in three extension courses, which are associated to promote the teaching of programming logic for elementary school students, with practical methodologies and propositions of challenges. The results of the experiments allowed the introduction of computational thinking, implementation of algorithms and basic notions of programming in a playful way, application development, teamwork in the assembly and programming of robots.
\end{abstract}

Keywords - Learning-by-doing; Programming Logic Teaching; Computational thinking; Ludification.

Resumo - Uma nova conjuntura educacional vem sendo trilhada devido a evolução da tecnologia e pesquisas demonstram a necessidade de novas abordagens didáticas para o ensino de programação de computadores. O objetivo principal deste estudo é apresentar as abordagens aplicadas em três cursos de extensão, que associam-se para promover o ensino da lógica de programação para alunos do ensino fundamental, com metodologias práticas e proposição de desafios. Os resultados das experiências permitiram a introdução do pensamento computacional, implementação de algoritmos e noções básicas de programação de uma maneira lúdica, desenvolvimento de aplicativos, trabalho em equipe na montagem e programação dos robôs.

Palavras-chave - Learning-by-doing; Ensino de lógica de programação; Pensamento computacional; Ludificação.

\section{INTRODUÇÃO}

Com o gradual aumento da importância da área de informática e das grandes transformações que estão ocorrendo, como por exemplo, a $4^{\text {a }}$ Revolução Industrial que traz vários outros termos, tais como: Inteligência Artificial, Big Data, Segurança da Informação, Internet das Coisas, Controle e Automação, Realidade Virtual e Aumentada, Robótica, Modelagem e Impressão 3D e entre outros.

Essas transformações influenciam diretamente nos processos de ensino e aprendizagem, estabelecem novos métodos e paradigmas relacionados à Educação 4.0, tais como, a Robótica Educacional, Problem-Based Learning, Pensamento Computacional (PC), Aprendizagem Criativa e Colaborativa e Metodologias Ativas. Ressalta-se que essas abordagens estão profundamente vinculadas à lógica de programação, que são essenciais para essa nova conjuntura educacional.

De acordo com Oliveira et. al [1] "é fundamental que as pessoas possam ter conhecimentos básicos de Computação desde o início da vida escolar, [...], embora seja também onde se encontram as principais dificuldades de aprendizagem”. Segundo Kafai [2] a lógica de programação deveria andar junto com outras disciplinas do ensino básico, pois o ensino de programação para crianças pode desenvolver o Pensamento Computacional e os passos lógicos para a resolução automatizada de problemas.

Além disso, Wangenheim et al. [3] declara que a programação de computadores é essencial para a computação, necessitando assim a aprendizagem da competência para desenvolver software. Também destacam que por meio do aprendizado de programação pode-se estimular a aprendizagem do Pensamento Computacional, que é uma abordagem para resolver problemas de maneira computacional envolvendo um conjunto de conceitos, tais como, abstração, iteração, recursão, entre outros.

Segundo Wing [4] o Pensamento Computacional é um processo de pensamento que envolve a criação de problemas e das suas soluções com base nos fundamentos da Ciência da Computação, pensamento crítico e define uma metodologia para resolver problemas.

Considerando esse contexto, o principal objetivo do trabalho é apresentar evidências práticas e impactos do ensino introdutório do Pensamento Computacional no ensino fundamental, por meio de um conjunto de cursos de extensão com a metodologia learning-by-doing no ensino de raciocínio lógico para computação.

\section{FUNDAMENTAÇÃO TEÓRICA E TRABALHOS RELACIONADOS}

O processo de ensino e aprendizagem de lógica de programação vem sendo amplamente pesquisado e novas técnicas e práticas são conduzidas em relação à antecipação desse conteúdo para o ensino fundamental II e médio de maneira introdutória, com o foco em desenvolver o raciocínio lógico de programação com ferramentas e técnicas lúdicas, criativas e práticas.

No projeto de extensão utilizou-se o framework de avaliação do pensamento computacional definido por Brennan e Resnick [5] para compreender as práticas de 
criador-em-ação e envolve três dimensões de competências: (i) Conceitos computacionais: são os conceitos que os projetistas empregam ao programar aborda sete conceitos que são amplamente úteis em projetos de $S_{c r a t c h}{ }^{1}$ e podem ser aplicados no contexto de programação, tais como, comandos sequenciais, comandos de repetição, eventos, paralelismo, comandos condicionais, operadores e dados; (ii) Práticas computacionais: são as práticas que os projetistas desenvolvem enquanto programam, estão concentradas nos processos de pensar e de aprender, como, interatividade, incremento, testes e depuração, reúso, abstração e modularidade; e, (iii) Perspectivas computacionais: são as perspectivas que os designers formam sobre o mundo ao seu redor e sobre si mesmos durante a programação: autoexpressão, conectividade e questionamento.

Diversos trabalhos e pesquisas sobre o assunto são elaboradas, tal como, o Oliveira et al. [1] onde os autores realizaram um curso para uma turma de 20 alunos do $9^{\circ}$ ano do ensino fundamental de escolas públicas, o qual envolveu os principais conteúdos de lógica de programação. As aulas foram ministradas por didática teórica/prática, onde os estudantes foram estimulados a desenvolver habilidades tanto por descoberta, quanto por meio de atividades direcionadas pelos ministrantes. Os autores concluem que "o conhecimento em computação é tão importante quanto qualquer outro encontrado nas ciências mais tradicionais" [1].

No trabalho de Duarte et al. [6] utilizou-se o Scratch como ferramenta de ensino-aprendizagem para estabelecer a importância da lógica de programação. O curso foi aplicado em duas escolas: uma pública, contando com alunos do $7^{\circ}$ ano; e outra particular, contando com alunos do $6^{\circ}, 7^{\circ}$ e $8^{\circ}$ ano. Com isso identificou-se que 93,75\% dos alunos possuem interesse por tecnologia, não obstante, $77 \%$ deles não havia participado de nenhum curso de informática, além disso, o Scratch foi bem aceito pelos alunos com uma taxa de $85,7 \%$ de aprovação. Os alunos demonstraram grande interesse pela dinâmica utilizada e foram notado a melhora em vários quesitos avaliados durante as oficinas.

O trabalho desenvolvido por Montoanelli et al. [7] ofertou um curso que utilizou as atividades do code.org para mensurar o impacto do curso no aprendizado. Aplicou-se um questionário para identificar a frequência do uso da tecnologia no cotidiano dos alunos e medir o grau de satisfação do curso. Evidenciou-se que $60 \%$ dos participantes acessam diariamente redes sociais, $80 \%$ ficaram satisfeitos com o que foi aprendido no curso e $84 \%$ dos estudantes utilizavam diariamente um computador, tablet ou celular. Assim, o projeto contribuiu mudando a perspectiva dos alunos sobre como explorar a computação de maneira lúdica para construir conhecimento, expressar e concretizar ideias por meio de um computador.

\section{Metodologia}

O público alvo foram alunos do oitavo e nono ano do ensino fundamental, predominantemente de colégios

\footnotetext{
${ }^{1}$ Scratch - Disponível em: scratch.mit.edu. acessado em: 28 out. 2020.
}

públicos, os cursos foram conduzidos pelo professor orientador juntamente com os alunos do curso Técnico em Informática Integrado ao Ensino Médio do IFPR Instituto Federal do Paraná Campus Quedas do Iguaçu que colaboraram para a sua realização como monitores e foram iniciados nas atividades de lecionar conteúdos de computação.

Na Figura 01, apresenta-se o mapa mental dos conteúdos dos cursos. O aprendizado é iniciado pelo curso Joglog, seguindo pelo DevAppLog e finalizado com o curso RobotLog, ou seja, os alunos participaram de três cursos em sequência.

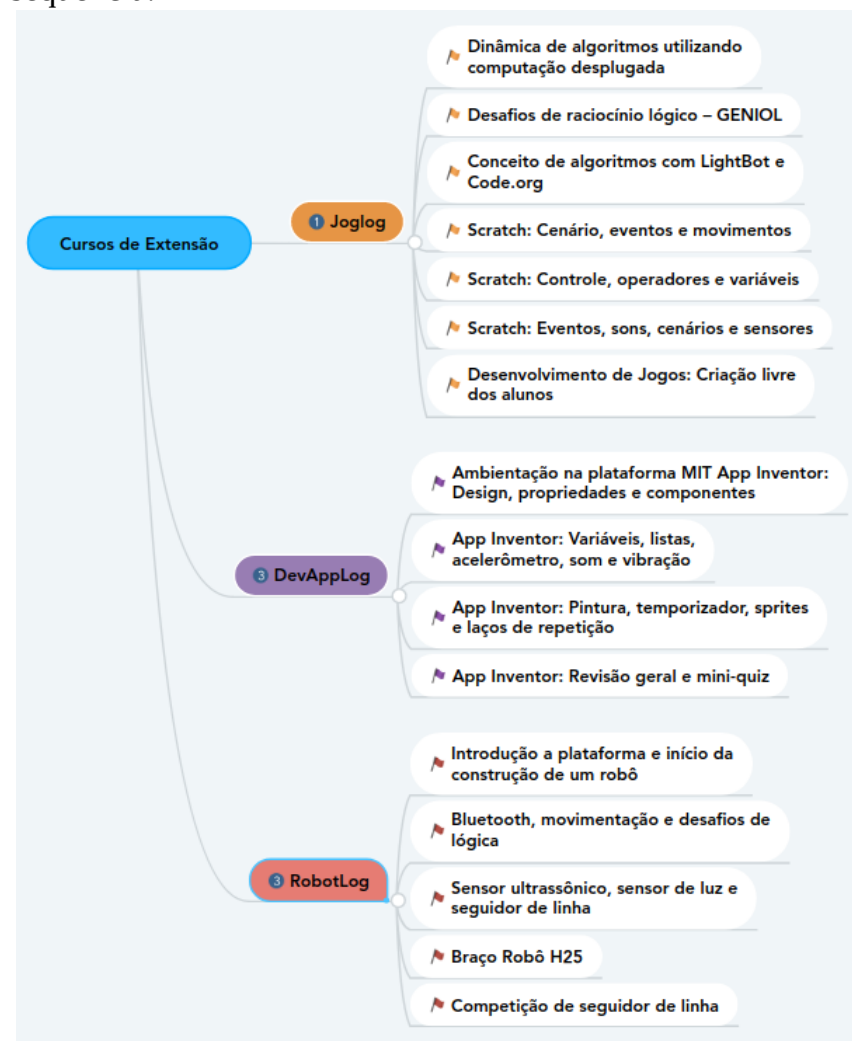

Figura 01. Conceitos, atividades e competências utilizadas nos cursos de extensão.

O projeto foi dividido em três cursos de extensão: JogLog, DevAppLog e RobotLog e abordou a metodologia learning-by-doing. Além disso, os cursos utilizam programação em blocos para minimizar as possíveis dificuldades com o entendimento de lógica de programação e sintaxe de linguagem de programação.

As aulas eram semanais durante a tarde e tendo a duração de três horas e por questões didáticas, eram dividas em dois períodos, durante o primeiro período eram abordados os conteúdos teóricos, onde os monitores apresentavam as atividades a serem trabalhadas, juntamente com a resolução de exemplos e no segundo período, os alunos resolviam as atividades propostas com apoio dos monitores. Na sequência a resolução dessas atividades eram explicadas pelos alunos para toda a turma.

O primeiro curso de extensão aplicado foi o JogLog que utilizou a plataforma Scratch. O Scratch é um projeto do grupo Lifelong Kindergarten no Media Lab do MIT, 
desenvolvido especialmente para idades entre 8 e 16 anos. A plataforma aborda várias habilidades essenciais, tais como, trabalho em equipe, pensamento criativo e aptidão a desenvolver software [8]. O curso ocorreu durante 13 semanas e inicialmente 34 alunos participaram.

No curso JogLog os alunos criaram seus jogos com a orientação dos monitores e apoio do professor, os alunos apresentaram e compartilharam com a turma os jogos, demonstrando conectividade. Durante a apresentação foi realizada a avaliação principal do desempenho dos alunos com base nas competências do Pensamento Computacional definidas por [5]. Os alunos aplicaram os blocos de Scratch: operadores, variáveis, controle, cenários, atores, eventos, movimentos, aparência e sensores.

Durante o DevAppLog a plataforma utilizada foi o MIT App Inventor ${ }^{2}$, a qual visa proporcionar para pessoas de todas as idades, a criação de aplicativos para smartphones e tablets [9]. A plataforma é open source e conta com mais de 6 milhões de usuários registrados. Ao final de sua realização cada aluno desenvolveu o seu próprio aplicativo, podendo testá-los em seu celular ou em tablets fornecidos pelo campus. O curso começou com 20 alunos e teve a duração de 4 semanas. Desde o primeiro encontro os alunos iniciaram o desenvolvimento de aplicativos utilizando blocos e aplicaram os principais elementos do App Inventor, tais como, design, propriedades, componentes, variáveis, listas, acelerômetro, som, vibração, pintura, temporizador, sprites e laços de repetição, e no final cada aluno desenvolveu seus aplicativos estilo quiz, envolvendo conhecimento de outras disciplinas, tais como, conhecimentos gerais, geografia, história e atualidades.

O terceiro curso realizado foi RobotLog que utilizou kits LEGO Mindstorms ${ }^{\circledR}$ EV3 para a criação de robôs. Os alunos foram separados em equipes de quatro pessoas, cada equipe recebeu um kit. Durante o decorrer das aulas vários projetos foram montados, tais como, robô para identificação da distância até objetos, braço robótico e ao final cada equipe montou seu próprio robô seguidor de linha para uma competição. Inicialmente com 11 alunos, o curso teve a duração de 5 semanas. Ressalta-se que o foco aqui foi no ensino apresentado de maneira inclusiva e livre.

\section{Resultados e Discussões}

Para verificar o nível de compreensão dos alunos nos conteúdos a serem abordados nos cursos de extensão, identificando a bagagem teórica e prática dos alunos, aplicou-se um questionário de caracterização de perfil. Foi possível observar que $87,88 \%$ dos alunos não tiveram nenhuma disciplina exclusiva de informática no ensino fundamental, e que $96,97 \%$ não sabiam o que é algoritmo.

Nas questões abertas do questionário de caracterização referente às definições dos conteúdos, identificou-se que $87,88 \%$ dos alunos não tinham conhecimento sobre comandos condicionais e 93,94\% não tinham conhecimento sobre lógica. Na avaliação final, 90,5\% dos alunos responderam corretamente sobre os conceitos de algoritmo

\footnotetext{
${ }^{2}$ AppInventor - Disponível em: appinventor.mit.edu. acessado em: 28 jun. 2020 .
}

e 87,5\% dos alunos obteve ótimo desempenho nos projetos finais do curso de extensão JogLog.

Em uma análise individual do curso de extensão JogLog verificou-se por meio da avaliação dos projetos finais, que constituiu no desenvolvimento de um jogo, o domínio das competências computacionais definidas por Brennan e Resnick [5] em três dimensões: Conceitos Computacionais, Práticas Computacionais e Perspectivas Computacionais. Referente a compreensão plena dos Conceitos Computacionais, $82,14 \%$ dos alunos demonstraram ter domínio da sua aplicação, em relação às Práticas Computacionais os resultados obtidos foram de 70,83\% de entendimento pleno e $80,56 \%$ nas Perspectivas Computacionais, ressalta-se que os demais alunos obtiveram domínio satisfatório e que nenhum obteve desempenho insatisfatório, essa avaliação foi realizada pelos 5 monitores e pelo professor.

Na avaliação dos alunos no curso de extensão DevAppLog verificou-se o domínio das competências computacionais e raciocínio lógico adquiridos no curso anterior, visto que os conteúdos são similares, porém o aprendizado acontece em uma nova proposta de aplicação. Nesse curso os alunos avançaram na compreensão de raciocínio lógico, resolução de problemas e estimularam a criatividade desenvolvendo seus próprios aplicativos.

Observou-se nos aplicativos desenvolvidos que os alunos aplicaram de maneira correta os principais conceitos e elementos de lógica de programação, tais como, eventos, controle, expressões lógicas e matemáticas, listas, variáveis e procedimentos. A avaliação do conhecimento obtido por cada aluno foi verificada por meio da arguição dos projetos, onde cada aluno explicou detalhadamente como desenvolveu o aplicativo. De acordo com as quatro etapas do pensamento computacional, $92,86 \%$ dos alunos obtiveram aprendizado pleno quanto a capacidade de decomposição, que é dividir o problema em outros menores, $78,57 \%$ dos alunos mostraram-se capazes de identificar padrões nos problemas, aplicar a abstração da solução e verificar se a solução pode ser válida para outros problemas e $85,71 \%$ dos alunos evidenciaram capacidade de construção de algoritmos.

No Curso de extensão RobotLog estimulou-se a motivação, engajamento e o trabalho em equipe, além da aplicação do raciocínio lógico para resolução dos desafios utilizando robôs. Os alunos obtiveram conhecimento sobre os principais componentes de robótica, tais como, sensores ultrassônicos, sensores de distância, sensores de cor, motores e bluetooth, aprenderam realizar movimentação, construíram um braço robótico e um robô seguidor de linha. Essas atividades práticas tiveram como objetivo consolidar os conceitos de lógica de programação e os três grupos concluíram de maneira efetiva todas as atividades propostas.

Em um panorama geral dos três cursos de extensão, observou-se que a maioria dos alunos demonstram, por meio de aplicação prática, a compreensão dos conceitos computacionais, principalmente os relacionados com 0 raciocínio lógico e lógica de programação, pois os cursos proporcionam a fixação dos conteúdos de maneira diferente e aplicada. Nota-se que os alunos adquiriram capacidade para elaborar um design e fazer uma construção evolutiva, 
descrevendo ciclos iterativos de imaginação, além disso, apresentaram estratégias e práticas para encontrar erros e defeitos nos seus próprios projetos.

A principal dificuldade enfrentada foi a desistência dos alunos durante os cursos. O JogLog iniciou com 34 alunos, porém 80\% concluíram, o DevAppLog obteve 20 alunos inscritos, entretanto na primeira aula estiveram presentes apenas 14 pessoas e o curso terminou com uma média de $78,5 \%$ dos alunos e o RobotLog foi mais consistente, com exceção de uma aula, durante todo o curso 11 alunos estiveram presentes.

Algumas questões podem ter influenciado na desistência dos alunos, como a localização do campus, então a maioria dos alunos dependia de transporte particular. Outro motivo pode ser as plataformas utilizadas, principalmente o MIT App Inventor, pois surgiram alguns comentários sobre a sua utilização ser complicada.

\section{CONSIDERAÇÕES FINAIS}

O projeto está em andamento e o conjunto de cursos de extensão JogLog, DevAppLog e RobotLog associam-se para capacitar os estudantes em competências computacionais, principalmente as vinculadas ao pensamento computacional em três abordagens, com atividades diversificadas, integrando o aprendizado de lógica de programação de maneira lúdica, intuitiva e atrativa.

A introdução dos estudantes em programação de computadores deve ter um processo que agregue um aprendizado positivo e criativo, para que os alunos não tenham um impacto negativo na transição para linguagem de programação e seguir na área de computação, considera-se que 16 alunos egressos do projeto ingressaram no curso Técnico em Informática do campus.

A integração das ferramentas digitais de suporte ao ensino e os métodos utilizados nos cursos relacionados com a construção de algoritmos, tendem a proporcionar um engajamento, motivação e despertar o interesse dos estudantes, desde os primeiros contatos com as atividades dos cursos, pois eles já pensam no desenvolvimentos dos seus projetos nos primeiros encontros.

O Curso JogLog permitiu a introdução do Pensamento Computacional, implementação de algoritmos e noções básicas de programação de computadores de uma maneira lúdica. No curso DevAppLog os alunos reforçam e complementam os conceitos e os aplicam integrando os elementos visuais do desenvolvimento de aplicativos. No curso de RobotLog os alunos desenvolvem raciocínio lógico por meio da montagem e programação de robôs, considerando os desafios propostos, esse curso concentra-se na engenhosidade, criatividade e trabalho colaborativo, pois todas as atividades são desenvolvidas em equipe, além disso, o grau de dificuldade na compreensão de lógica de programação diminuiu consideravelmente após os conhecimentos adquiridos nos cursos anteriores.

O relato de experiência apresentado nesse artigo evidência a eficácia da aplicação das abordagens introdutórias ao pensamento computacional, competências computacionais, construção de algoritmos, e contribui para a disseminação e para a inclusão da aprendizagem de lógica de programação no ensino fundamental, de maneira lúdica, criativa e dinâmica.

\section{Agradecimentos}

Agradecemos ao Instituto Federal do Paraná - IFPR pelo auxílio financeiro por meio de bolsa estudantil da Diretoria de Extensão, Arte e Cultura - DIEXT/PROEPPI e aos monitores voluntários dos cursos de extensão.

\section{REFERÊNCIAS}

[1] M. L. S. Oliveira, A. A. Souza, A. F. Barbosa, e E. F. S. Barreiros, "Ensino de lógica de programação no ensino fundamental utilizando o scratch: um relato de experiência”, in Proceedings of XXXIV Congresso da Sociedade Brasileira de Computação (CSBC’14), 2014, p. 1493-1502.

[2] Y. B. Kafai e Q. Burke, “Computer Programming Goes Back to School”, Phi Delta Kappan, vol. 95, no 1, p. 61-65, set. 2013.

[3] C. G. V. Wangenheim, V. R. Nunes, e G. D. Santos, "Ensino de Computação com SCRATCH no Ensino Fundamental - Um Estudo de Caso”, RBIE - Revista Brasileira de Informática na Educação, vol. 22, no 03, p. 115, nov. 2014.

[4] J. M. Wing, "Computational thinking”, Communications of the ACM, vol. 49, no 3, p. 33-35, 2006.

[5] K. Brennan e M. Resnick, "New frameworks for studying and assessing the development of computational thinking”, in Proceedings of the 2012 annual meeting of the American Educational Research Association, Vancouver, Canada, 2012, p. $1-25$.

[6] K. Duarte, T. Silveira, e M. Borges, “Abordagem para o Ensino da Lógica de Programação em Escolas do Ensino Fundamental II através da Ferramenta Scratch 2.0”, Recife, Pernambuco, Brasil, out. 2017, p. 175, doi: 10.5753/cbie.wie.2017.175.

[7] G. H. Montoanelli, J. P. Rolim, F. F. F. Peres, C. R. M. Mauricio, E. N. Pereira, e R. C. Silva, "Ensino de Programação nas Escolas: Um relato de experiência”, in Anais do Congresso Latino-Americano de Software Livre e Tecnologias Abertas (Latinoware 2019), Brasil, nov. 2019, p. 40-45.

[8] M. Resnick et al., "Scratch: programming for all", Communications of the ACM, vol. 52, no 11, p. 60-67, nov. 2009, doi: 10.1145/1592761.1592779.

[9] E. W. Patton, M. Tissenbaum, e F. Harunani, "MIT App Inventor: Objectives, Design, and Development”, in Computational Thinking Education, S.-C. Kong e H. Abelson, Orgs. Singapore: Springer Singapore, 2019, p. 31-49. 